\title{
What Echocardiographic Measure Should Be Used to Assess Right Ventricular Function in Tetralogy of Fallot?
}

\section{Jill J Savla, Valerie De Matteo, Yan Wang and Laura Mercer-Rosa*}

Division of Cardiology, University of Pennsylvania, Perelman School of Medicine, Children's Hospital of Philadelphia, USA

\section{Description}

The assessment of right ventricular (RV) function in patients with congenital heart disease is the focus of significant research and clinical interest. The RV's anterior position in the chest, complex geometric shape, and unique pattern of contractility make the assessment of RV function by conventional 2-dimensional parameters challenging [1-3]. In Tetralogy Of Fallot (TOF) in particular, investigators have studied multiple non-invasive measures to evaluate the RV's systolic function, including Tricuspid Annular Plane Systolic Excursion (TAPSE), Fractional Area Change (FAC), isovolumic acceleration time, tissue doppler-derived tricuspid systolic velocity (S'), longitudinal peak systolic strain, stress echocardiography, and more recently threedimensional echocardiography [4-6]. Although the use of these methods has been suggested in particular for the follow up of patients with TOF, pulmonary regurgitation, progressive RV dilation, and dysfunction, the clinical utility of certain parameters remains undefined.

When deciding how to approach the assessment of RV function in TOF by echocardiography, which measures should be reported for clinical use? The American Society of Echocardiography has proposed a variety of diagnostic imaging modalities for the evaluation of patients with TOF, with a focus on older patients with repaired TOF [4]. This report suggests the complementary use of different modalities to assess RV function, all of which have inherent limitations. However, we propose a few specific methods to be used in clinical practice. Fractional area change (FAC) is measured in squared centimeters $\left(\mathrm{cm}^{2}\right)$ on a modified apical 4 -chamber view by 2 -dimensional echocardiography, but can also be measured using speckle-tracking software. First, the area of change is traced along the RV endocardium during both diastole and systole. Second, the RV diastolic area is subtracted by the RV systolic area, and then divided by the RV diastolic area, to produce the FAC calculation. This is a relatively easy and reproducible parameter that has at least modest correlation with cardiac magnetic resonance imaging (CMR) data [7-9], FAC should be utilized more often, especially given the limitations of other 2-dimensional parameters (such as TAPSE) in patients with structurally-abnormal or surgically-altered right ventricles, such as TOF $[10,11]$.

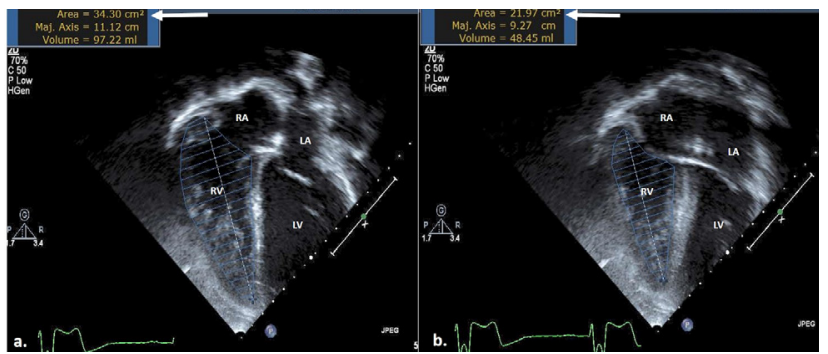

FAC: Fractional Area Change; RA: Right Atrium; RV: Right Ventricle; LA: Left Atrium; LV: Left Ventricle.

Figure 1: Fractional area change measurement obtained during end-diastole (a) and end-systole (b) in the right ventricle of a patient with tetralogy of Fallot. The arrows demonstrate the measured areas during diastole and systole. The calculated FAC $[(34.3-21.97) / 34.3]=35.9 \%$.

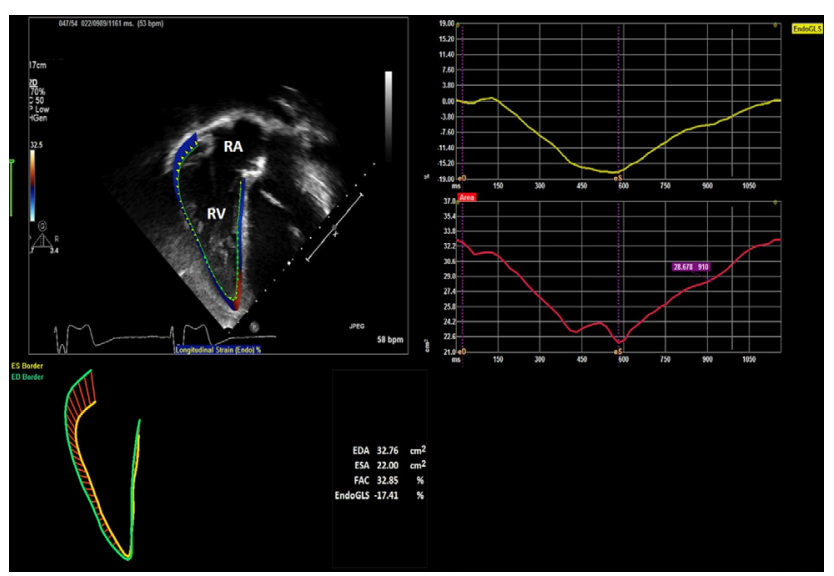

Figure 2: Right ventricle fractional area change obtained with $\mathrm{TomTec}^{\mathrm{TM}}$ software. RA, right atrium; RV, right ventricle.

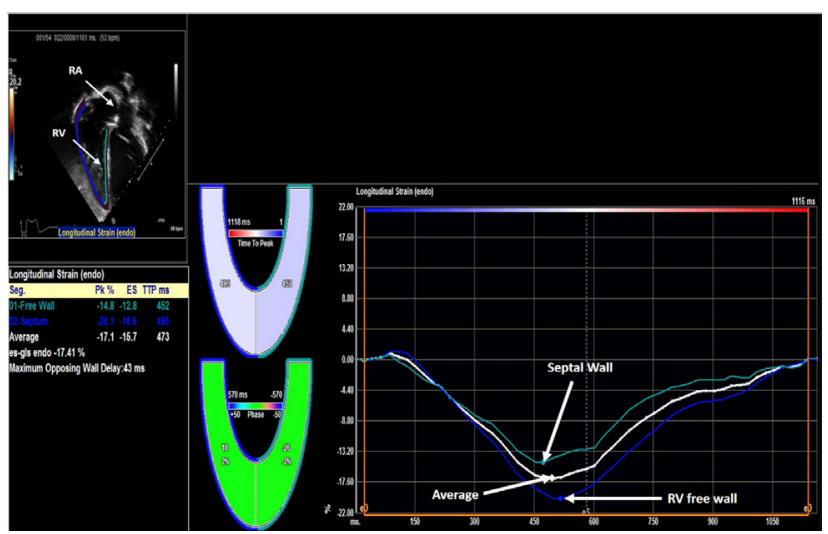

Figure 3: Right ventricle global longitudinal strain (TomTec ${ }^{\mathrm{TM}}$ ). The curves demonstrate segmental wall motion of the right ventricular septal and free walls. $\mathrm{RA}$, right atrium; $\mathrm{RV}$, right ventricle.

Recent studies have shown that myocardial deformation is applicable to patients with TOF. Global longitudinal RV strain is a measure of intrinsic myocardial contractility that calculates cardiac deformation as a percentage change in length [12-14]. It is quantitative,

*Corresponding author: Laura Mercer-Rosa, Division of Cardiology, University of Pennsylvania, Perelman School of Medicine, Children's Hospital of Philadelphia, USA, Tel: 215-590-4040; E-mail: mercerrosal@email.chop.edu

Received July 04, 2017; Accepted July 12, 2017; Published July 14, 2017

Citation: Savla JJ, De Matteo V, Wang Y, Mercer-Rosa L (2017) What Echocardiographic Measure Should Be Used to Assess Right Ventricular Function in Tetralogy of Fallot? J Cardiovasc Dis Diagn 5: 287. doi: 10.4172/2329-9517.1000287

Copyright: @ 2017 Savla JJ, et al. This is an open-access article distributed under the terms of the Creative Commons Attribution License, which permits unrestricted use, distribution, and reproduction in any medium, provided the original author and source are credited. 
angle-independent, and less dependent on loading conditions compared to other echocardiographic measures, which is a particularly convenient characteristic when dealing with volume loaded RVs in patients with TOF [15-17]. After training personnel and achieving adequate reproducibility, global longitudinal RV strain appears to be a sensitive measure of ventricular function, as evidence suggests that strain is affected prior to changes in other more blunt measures of function [18]. Therefore, strain is the next parameter that should be added to the clinical echocardiography report. At present, and until normative data are well established and the variability among vendors is reduced, we suggest its use primarily in the follow-up of individual patients over time, where changes in RV peak systolic longitudinal strain may precede overt changes in ejection fraction and therefore alert the clinician [19].

Examples of these specific methods being utilized in clinical practice are shown in the accompanying Figures 1-3 from an adolescent male with TOF and absent pulmonary valve, who underwent initial surgical repair with a conduit from the right ventricle to the pulmonary artery and closure of the ventricular septal defect in infancy, followed by conduit replacement at 12 years of age. As a medical community with interest in improving outcomes for patients with TOF, our collective goal should be to better understand RV mechanics, apply the best imaging tools to accurately quantitate RV function, identify patients at increased risk who may require more frequent monitoring or earlier intervention, predict clinical or procedural outcomes, and ultimately provide the scientific evidence that guides the care and treatment of our patients.

\section{References}

1. Lopez L, Colan SD, Frommelt PC, Ensing GJ, Kendall K, et al. (2010) Recommendations for quantification methods during the performance of a pediatric echocardiogram: A report from the pediatric measurements writing group of the American Society of Echocardiography Pediatric and Congenital Heart Disease Council. J Am Soc Echocardiogr 23: 465-495.

2. Rudski LG, Lai WW, Afilalo J, Hua L, Handschumacher MD, et al. (2010) Guidelines for the echocardiographic assessment of the right heart in adults: A report from the American Society of Echocardiography. J Am Soc Echocardiogr 23: $685-713$

3. Hamilton-Craig CR, Stedman K, Maxwell R, Anderson B, Stanton T, et al. (2016) Accuracy of quantitative echocardiographic measures of right ventricula function as compared to cardiovascular magnetic resonance. Int $\mathrm{J}$ Cardiol Heart Vasc 12: 38-44.

4. Valente AM, Cook S, Festa P, Helen Ko H, Krishnamurthy R, et al. (2014) Multimodality imaging guidelines for patients with repaired tetralogy of Fallot A report from the American Society of Echocardiography developed in collaboration with the society for cardiovascular magnetic resonance and the society for pediatric radiology. J Am Soc Echocardiogr 27: 111-141.

5. Raj R, Puri GD, Jayant A, Singh Thingnam SK, Sandip Singh R, et al. (2016)
Perioperative echocardiography-derived right ventricle function parameters and early outcomes after tetralogy of Fallot repair in mid-childhood: A singlecenter, prospective observational study. Echocardiography 33: 1710-1717.

6. Roche SL, Grosse-Wortmann L, Friedberg MK, Redington AN, Stephens D, et al. (2015) Exercise echocardiography demonstrates biventricular systolic dysfunction and reveals decreased left ventricular contractile reserve in children after tetralogy of Fallot repair. J Am Soc Echocardiogr 28: 294-301.

7. Selly JB, Iriart X, Roubertie F, Mauriat P, Marek J, et al. (2015) Multivariable assessment of the right ventricle by echocardiography in patients with repaired tetralogy of Fallot undergoing pulmonary valve replacement: A comparative study with magnetic resonance imaging. Arch Cardiovasc Dis 108: 5-15.

8. Okada DR, Rahmouni HW, Herrmann HC, Bavaria JE, Forfia PR, et al. (2014) Assessment of right ventricular function by transthoracic echocardiography following aortic valve replacement. Echocardiography 31: 552-557.

9. Lopez-Candales A, Dohi K, Rajagopalan N, Gulyasy B, Bazaz R, et al. (2008) Defining normal variables of right ventricular size and function in pulmonary hypertension: An echocardiographic study. Postgrad Med J 84: 40-45.

10. Mercer-Rosa L, Parnell A, Forfia PR, Yang W, Goldmuntz E, et al. (2013) Tricuspid annular plane systolic excursion in the assessment of right ventricular function in children and adolescents after repair of tetralogy of fallot. J Am Soc Echocardiogr 26: 1322-1329.

11. Avitabile CM, Whitehead K, Fogel M, Mercer-Rosa L (2014) Tricuspid annular plane systolic excursion does not correlate with right ventricular ejection fraction in patients with hypoplastic left heart syndrome after fontan palliation. Pediatr Cardiol 35: 1253-1258.

12. Sutherland GR, Di Salvo G, Claus P, D'hooge J, Bijnens B (2004) Strain and strain rate imaging: A new clinical approach to quantifying regional myocardial function. J Am Soc Echocardiogr 17: 788-802.

13. Horton KD, Meece RW, Hill JC (2009) Assessment of the right ventricle by echocardiography: A primer for cardiac sonographers. J Am Soc Echocardiogr 22: 776-792.

14. Gorcsan J, Tanaka H (2011) Echocardiographic assessment of myocardial strain. J Am Coll Cardiol 58: 1401-1413.

15. Li Y, Xie M, Wang X, Lu Q, Zhang L, et al. (2015) Impaired right and left ventricular function in asymptomatic children with repaired tetralogy of Fallot by two-dimensional speckle tracking echocardiography study. Echocardiography 32: $135-143$

16. Bernard Y, Morel M, Descotes-Genon V, Jehl J, Meneveau N, et al. (2014) Value of speckle tracking for the assessment of right ventricular function in patients operated on for tetralogy of fallot. Comparison with magnetic resonance imaging. Echocardiography 31: 474-482.

17. Tori KD, Soriano BD, Buddhe S (2016) Right ventricular global longitudinal strain in repaired tetralogy of Fallot. Echocardiography 33: 1557-1562.

18. Mondillo S, Galderisi M, Mele D, Cameli M, Lomoriello VS, et al. (2011) Speckle-tracking echocardiography: A new technique for assessing myocardial function. J Ultrasound Med 30: 71-83.

19. Scherptong RW, Mollema SA, Blom NA, Kroft LJ, De Roos A, et al. (2009) Right ventricular peak systolic longitudinal strain is a sensitive marker for right ventricular deterioration in adult patients with tetralogy of Fallot. Int $J$ Cardiovasc Imaging 25: 669-676. 